\title{
p73 participates in WWOX-mediated apoptosis in leukemia cells
}

\author{
DONGHONG LIN ${ }^{1 *}$, ZHAOLEI CUI ${ }^{1 *}$, LINGYING KONG $^{2}$, FENG CHENG $^{3}$, JIANPING XU ${ }^{1}$ and FENGHUA LAN ${ }^{4}$ \\ ${ }^{1}$ Department of Clinical Laboratory, Fujian Medical University, Fuzhou 35004; ${ }^{2}$ Department of Pathology, \\ Affiliated People's Hospital of Fujian Province, Fujian University of Tradition Chinese Medicine, Fuzhou 350004; \\ ${ }^{3}$ Department of Clinical Laboratory, Affiliated Second People's Hospital, Fujian University of Tradition \\ Chinese Medicine, Fuzhou 350003; ${ }^{4}$ Department of Clinical Genetics and Experimental Medicine, \\ Fuzhou General Hospital, Fujian Medical University, Fuzhou 350025, P.R. China
}

Received November 24, 2012; Accepted January 18, 2013

DOI: $10.3892 / \mathrm{ijmm} .2013 .1289$

\begin{abstract}
The WWOX gene is considered to be a tumorsuppressor gene which encodes a protein (Wwox) implicated in various types of solid human cancers. It has been shown that overexpression of WWOX in human tumors promotes apoptosis in vitro and suppresses tumor growth in vivo. Recently, we investigated the effects of WWOX overexpression in vitro and observed marked growth arrest in human leukemia cells; however, the underlying mechanism(s) for this effect is unknown. The present study aimed to elucidate the primary mechanism(s) underlying WWOX-mediated apoptosis in human leukemia. We traced the interactions between WWOX and its associated factors p73 and p53 after WWOX overexpression was induced in Jurkat and K562 cells. Our data revealed that $\mathrm{p} 73$ participates in WWOX-mediated apoptosis in Jurkat and K562 cells through binding with Wwox in the cytoplasm without a nuclear-cytoplasmic translocation.
\end{abstract}

\section{Introduction}

WWOX (WW domain containing oxidoreductase) is a tumorsuppressor gene located at 16q23.3-24.1, a chromosome region that spans the second most common human fragile site named FRA16D (1). The WWOX gene is composed of nine exons and encodes a 46-kDa Wwox protein that consists of two N-terminal WW domains and one C-terminal short-chain dehydrogenase domain named SDR (1-3). WW domains are characterized for their interactions with proline-containing ligands, and they

Correspondence to: Professor Donghong Lin, Department of Clinical Laboratory, Fujian Medical University, No. 88 Jiaotong Road, Taijiang, Fuzhou 350004, P.R. China

E-mail: lindh65@163.com; cuileidizi@163.com

*Contributed equally

Key words: WWOX, p73, overexpression, apoptosis, mechanism, leukemia play an important role in mediating protein-protein interactions (3). The SDR domain is located in the central region of Wwox, and contains amino acid sequence homology to the steroid oxidoreductases (3-5). It has been demonstrated that WWOX plays a functional role as a tumor-suppressor gene, as loss or alteration of WWOX was found in multiple types of solid cancers including breast, lung, esophagus, pancreas, and other cancers (6-10). When overexpressed, WWOX is capable of initiating apoptosis in vitro and suppresses tumor growth in vivo $(11,12)$. WWOX-knockout mice also showed a shortened lifespan or defects in bone metabolism or increased incidence of tumorigenesis, as well as other deficiencies $(11,13)$.

WWOX partners consist of an extensive scope including the apoptosis-associated factors p73 and p53 (14-18). Studies have reported that ectopic WWOX exhibits proapoptotic and tumor inhibitory functions, probably by interacting with p73 or p53 (17), and WWOX expression triggers redistribution of p73 from the nucleus to the cytoplasm. In addition, the proapoptotic activity of WWOX can be enhanced by cytoplasmic p73 (14). Gomes et al recently found that $W W O X$ mRNA levels are associated with p53 (18). Therefore, whether WWOX interacts with p73 or p53 in humans is not well validated. Thus, we investigated the effects of WWOX overexpression on the biological properties of Jurkat and K562 cells, and observed a dramatic growth arrest in these cell lines. To elucidate the underlying mechanisms, we investigated whether WWOX interacts with p73 or p53. We initially increased WWOX expression in Jurkat ( $W W O X$ mRNA and Wwox absent) and K562 (WWOX mRNA low expression and Wwox absent) cells by transfecting them with the pGC-FU-WWOX lentiviral plasmid, and then traced the interactions between WWOX and $\mathrm{p} 73$ or p53 via co-immunoprecipitation and western blot assays. We confirmed specific interactions between WWOX and p73 in human leukemia.

\section{Materials and methods}

Materials. Jurkat and K562 cells were purchased from the Chinese Academy of Sciences (Shanghai, China). The main reagents are listed as follows: RPMI-1640, FBS (Gibco-BRL, Carlsbad, CA, USA); TRIzol reagent, Lipofectamine 2000 (Invitrogen, USA); AgeI enzyme (NEB, USA); RT-PCR kit 
(Fermentas, USA); qPCR kit (Roche Diagonostics, USA); pGC-FU lentiviral vector, pHelper 1.0, pHelper 2.0, T4 DNA ligase (Genechem, Shanghai, China); rabbit anti-human Wwox, mouse monoclonal anti-human p53, anti-p73, antilamin B (Abcam, USA); RIPA lysis buffer, Co-IP kit, DAPI, mouse anti-human $\beta$-actin, FITC-conjugated anti-rabbit IgG, Cy3-conjugated anti-mouse IgG, mouse anti-human tubulin (Beyotime, Shanghai, China); nuclear and cytoplasmic protein extraction kit (Zoman Biotechnology, Beijing, China).

Cell culture. Jurkat and K562 cells were maintained in RPMI1640 supplemented with $10 \%$ fetal bovine serum, and cultured at $37^{\circ} \mathrm{C}$ in $5 \% \mathrm{CO}_{2}$.

Construction of the WWOX lentiviral vectors. The human WWOX gene was cloned from normal human liver via RT-PCR with the primer pairs: F, 5'-GAGGATCCCCGG GTACCGGTCGCCACCATGGCAGCGCTGCGCTAC-3'; R, 5'-TCACCATGGTGGCGACCGGGCCGGACTGGCTGCC AAG-3'. The PCR product was digested by AgeI enzyme, and the lentiviral vector (pGC-FU) was treated in the same manner. WWOX cDNA and pGC-FU were integrated with the aid of the T4 DNA ligase, followed by transformation, clone picking and amplification, respectively. The combined plasmids were extracted and sent for sequencing. Two types of package vectors (pHelper 1.0 and pHelper 2.0) were required to make the pGC-FU recombinant integrated. All vectors were propagated in 293T cells using Lipofectamine 2000.

Cell infection and cell growth assays. pGC-FU-WWOX (encoding Wwox-GFP fusion protein), pGC-FU-GFP (a mock plasmid only encoding GFP) and untreated cells (blank control) were established. Cells were seeded at a density of $1 \times 10^{5} / \mathrm{ml}$, and the lentiviral plasmid was added with an optimal MOI of 50 for Jurkat and MOI of 30 for K562 cells. A commercial Cell Counting Kit- 8 (Dojindo, Japan) was used to evaluate the growth-inhibition effects according to the specified protocol. The optical density (OD value proportional to the cell number) was measured with a microculture plate reader (Bio Tek Instruments, USA) at both 450 and $630 \mathrm{~nm}$.

Reverse transcription-PCR analysis. Total RNA was extracted with TRIzol reagent and reverse transcribed into cDNA. The target genes were then amplified with the following primers: WWOX (6-8 exons) F, 5'-CACGCATTTTAGAAGAATGG-3'; R, 5'-GACAGCAGCACAGTACACG-3'; GAPDH F, 5'-CAAG GTCATCCATGACAACTTTG-3'; R, 5'-GTCCACCACCC TGTTGCTGTAG-3'. PCR was performed using a PCR kit (Biomed, Beijing, China), and the amplifications were carried out in a Mastercycler gradient thermocycler (Applied Biosystems, USA) as follows: an initial denaturation for $5 \mathrm{~min}$ at $94^{\circ} \mathrm{C}$ followed by 30 cycles of $94^{\circ} \mathrm{C}$ for $30 \mathrm{sec}, 55^{\circ} \mathrm{C}$ for $30 \mathrm{sec}, 72^{\circ} \mathrm{C}$ for $30 \mathrm{sec}$, and a final extension for $7 \mathrm{~min}$ at $72^{\circ} \mathrm{C}$.

Flow cytometric analysis. The apoptosis ratio (\%) was assessed by flow cytometry (FCM). In brief, infected cells were collected and stained by Annexin V PE/7-aminoactinomycin D (KeyGEN Biotech, Nanjing, China) according the manufacturer's instructions, and analyzed using a Becton Dickinson FACSCalibur.
Immunofluorescence assay. Concisely, the cell monolayer was fixed with $4 \%$ paraformaldehyde, and incubated at $4^{\circ} \mathrm{C}$ overnight with rabbit anti-Wwox (1:500), mouse anti-p53 (1:300) and anti-p73 (1:300), respectively. FITC-conjugated anti-rabbit and Cy3-conjugated anti-mouse IgG all diluted at 1:1000. DAPI was used to dye the cell nuclei. Stained cells were washed by PBS and observed via fluorescence microscopy.

qPCR analysis. qPCR test was carried out with SYBR Green PCR Master Mix under the recommended conditions. Primer sequences were described as: p53 F, 5'-TGCAATAGGTGTG CGTCAGAA-3'; R, 5'-CCCCGGGACAAAGCAAA-3'; p73 F, 5'-AACGCTGCCCCAACCACGAG-3'; R, 5'-GCCGGTT CATGCCCCCTACA-3'; GAPDH F, 5'-GAAGGTGAAGGT CGGAGT-3'; R, 5'-GAAGATGGTGATGGGATTTC-3'. The comparative $\mathrm{Ct}$ method was used to calculate the relative expression level of p73 or p53 as compared with GAPDH.

Western blot and co-immunoprecipitation assays. A nuclear and cytoplasmic protein extraction kit was used for the isolation of proteins from the nucleus and cytoplasm of the cells. The primary antibodies and their dilutions used were: rabbit anti-Wwox (1:1000), mouse anti-p53 (1:1000), anti-p73 (1:500), anti- $\beta$-actin (1:1000), anti-lamin B (1:1000) and anti-tubulin (1:1000). Co-immunoprecipitation assay was carried out according to the protocol of a commercial Co-IP kit. Rabbit anti-Wwox $(2 \mu \mathrm{g})$ was added to protein $\mathrm{A} / \mathrm{G}$ agarose to hook p73 or p53, and for the mutual detection, $2 \mu \mathrm{g}$ mouse anti-p73 was used to hook Wwox or p53.

Statistical analysis. All data are expressed as means \pm standard deviation (SD). Differences between groups were analyzed by the Student's t-test or the non-parametric test using SPSS 13.0, and statistical significance for the data was set at $\mathrm{p}<0.05$.

\section{Results}

WWOX was successfully transfected into Jurkat and K562 cells. We initially examined whether WWOX cDNA was successfully transfected into Jurkat and K562 cells using fluorescence microscopy, RT-PCR and western blotting. The results revealed that cells infected with pGC-FU-WWOX and pGC-FU-GFP were all observed to express GFP at $48 \mathrm{~h}$ after infection (Fig. 1A). RT-PCR and western blot analysis determined that cells infected with pGC-FU-WWOX exhibited a high expression level of WWOX mRNA and Wwox protein when compared with cells transfected with pGC-FU-GFP (Fig. 1B), indicating that WWOX cDNA was successfully transfected into Jurkat and K562 cells.

WWOX overexpression reduces the viability of Jurkat and K562 cells. The effects of WWOX overexpression on the viability of Jurkat and K562 cells was assessed by CCK-8 assay. As shown in Fig. 2, at 24, 48, 72 and 96 h following transfection, the OD values (proportional to the cell numbers) of the pGC-FU-WWOX-infected Jurkat cells were 0.192 \pm 0.006 , $0.229 \pm 0.017,0.274 \pm 0.016$ and $0.349 \pm 0.020$, respectively. Regarding the K562 cells, the OD values were $0.596 \pm 0.017$, $0.787 \pm 0.06,0.746 \pm 0.039$ and $0.803 \pm 0.023$, respectively; significantly lower when compared with the OD value of the 
A

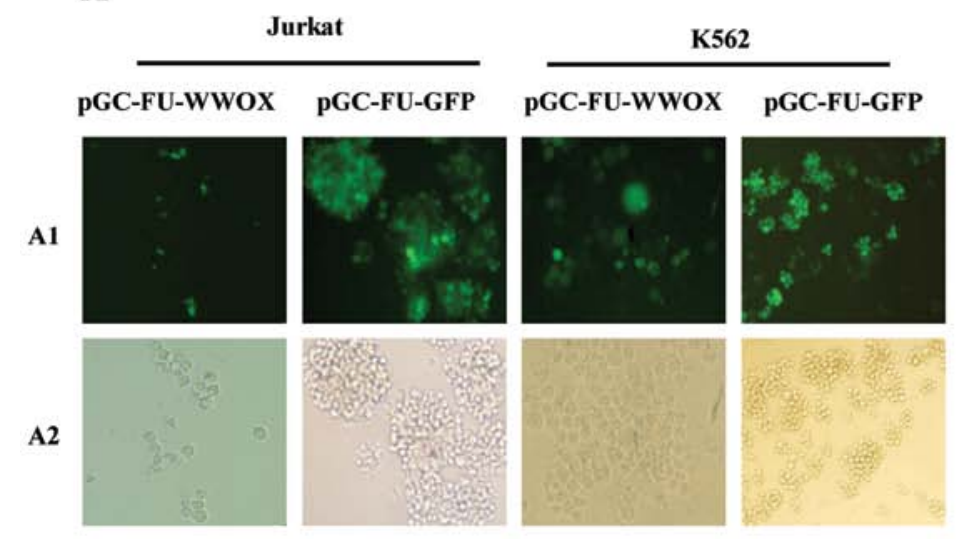

B

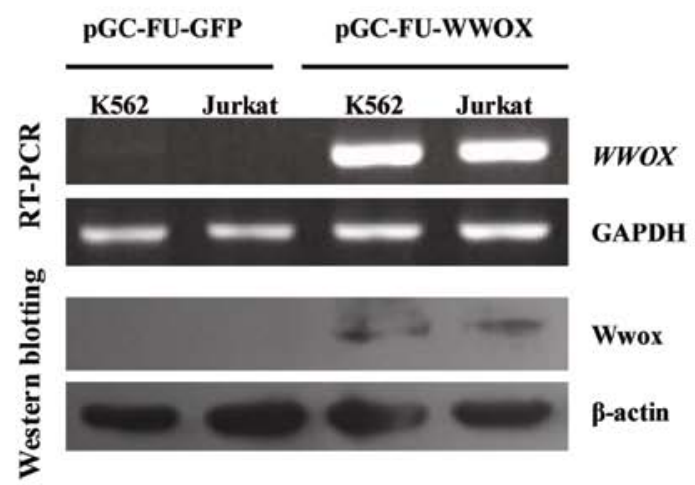

Figure 1. Expression of WWOX in Jurkat and K562 cells after infection. (A) Expression of Wwox-GFP and mock vector GFP was observed at 48 h after infection via fluorescence microscopy. A1, fluorescent light, x200; A2, ordinary light, x200. (B) Expression of WWOX in Jurkat and K562 cells after infection was detected by RT-PCR and western blotting.
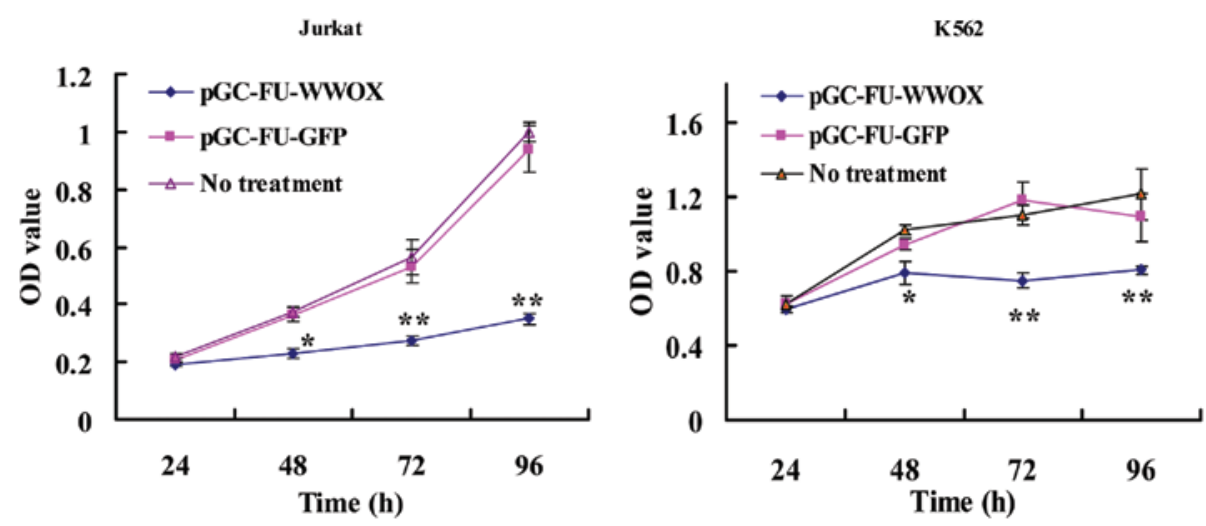

Figure 2. Effects of WWOX overexpression on the cell viability of Jurkat and K562 cells. A commercial Cell Counting Kit-8 was used to evaluate the effects of WWOX overexpression on the inhibition of viability. The OD value proportional to the cell number was measured and plotted on the the growth curve $\left({ }^{*} \mathrm{p}<0.05 ;{ }^{* *} \mathrm{p}<0.01\right.$, compared with the untreated cells).

untreated cells $(\mathrm{p}<0.05)$, while both Jurkat and K562 cells infected with pGC-FU-GFP exhibited no difference when compared with the untreated cells $(\mathrm{p}>0.05)$. This suggests that WWOX overexpression results in a reduction in the viability of Jurkat and K562 cells.

WWOX overexpression promotes apoptosis in Jurkat and K562 cells. To investigate whether the suppressive effects of WWOX overexpression on cell viability is due to apoptosis, we employed FCM after cells were stained with Annexin V $\mathrm{PE} / 7$-aminoactinomycin D. Cells infected with pGC-FUWWOX exhibited higher and increased apoptosis ratios (\%) when compared with the apoptosis ratio of cells infected with pGC-FU-GFP during time lapse. The apoptosis ratio (\%) of pGC-FU-WWOX-infected Jurkat cells was $18.29 \pm 1.62 \%$ at $48 \mathrm{~h}$, which increased to $84.15 \pm 1.10 \%$ at $96 \mathrm{~h}$. Regarding pGC-FU-WWOX-infected K562 cells, the apoptosis ratio was $18.57 \pm 1.30 \%$ at $48 \mathrm{~h}$, and increased to $93.59 \pm 1.26 \%$ at $96 \mathrm{~h}$ (all with $\mathrm{p}<0.05$ when compared with the pGC-FU-GFP-infected cells) (Fig. 3).

WWOX overexpression does not cause a change in or translocation of p73 and p53. To investigate whether WWOX overexpression causes a change in expression level or a translocation of p73 or p53 in Jurkat and K562 cells, we analyzed their subcellular locations via immunofluorescence assay. Wwox in the pGC-FU-WWOX-infected cells was located mainly in the cytoplasm, while p73 and p53 were also mainly located in the cytoplasm. pGC-FU-GFP-infected cells as well as the untreated cells exhibited similar locations for $\mathrm{p} 73$ and p53 when compared with the pGC-FU-WWOX-infected cells (Fig. 4A). We then assessed the changes in p73 and p53 at the mRNA and protein levels via qPCR and western blotting. qPCR results revealed that changes in p73 and p53 mRNA in the pGC-FU-WWOX-infected cells exhibited no difference when compared with the pGC-FU-GFP-infected cells ( $p>0.05)$ (Fig. 4B). Western blot assay demonstrated that total protein (even in different organelles) of p73 and p53 in the pGC-FUWWOX-infected cells was altered inconspicuously compared with the pGC-FU-GFP-infected cells ( $p>0.05$ ) (Fig. 4C and $\mathrm{D})$, indicating that WWOX overexpression was unable to cause a change in translocation of p73 and p53.

Ectopic Wwox binds with p73 instead of p53 in the cytoplasm. To investigate the interactions between WWOX and p73 or p53, co-immunoprecipitation and western blot analysis were 

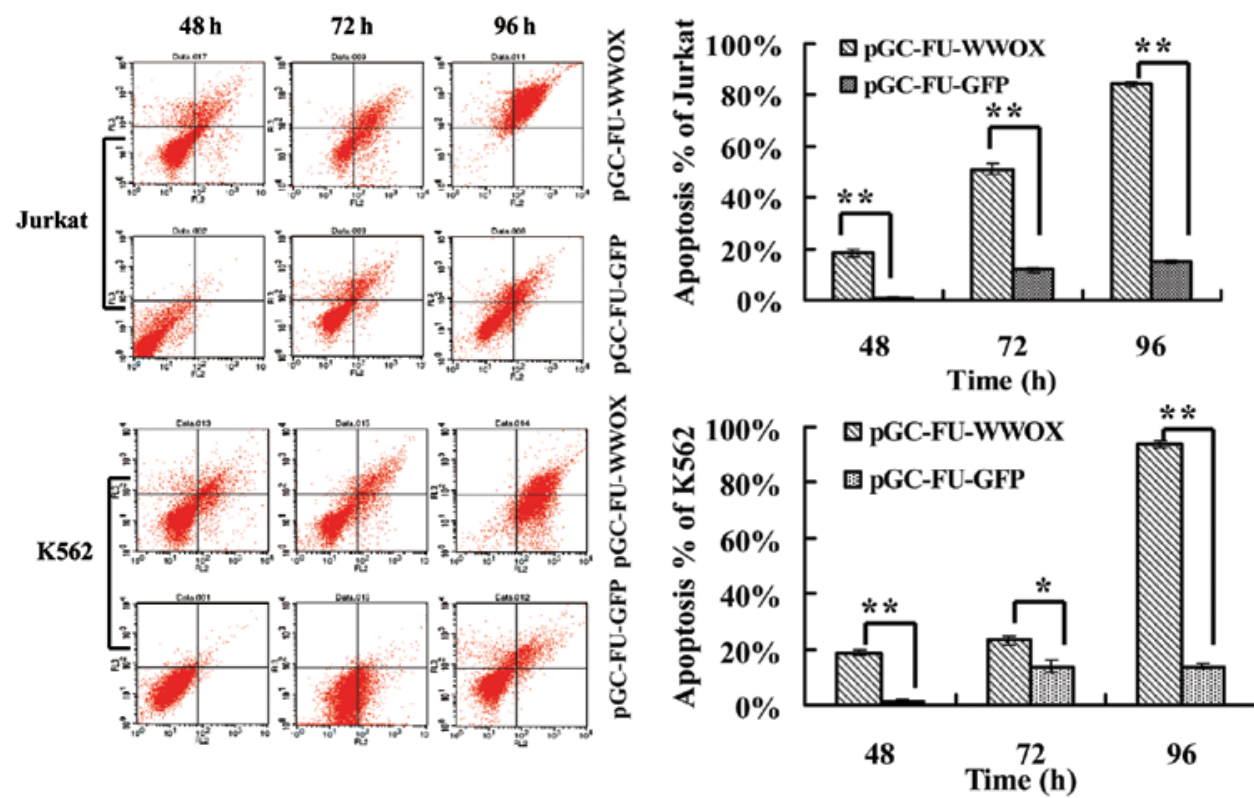

Figure 3. Effects of WWOX overexpression on the apoptosis ratio (\%) of Jurkat and K562 cells as assessed by flow cytometry (FCM). As the lentiviral vectors, pGC-FU-WWOX and pGC-FU-GFP, expressed GFP, we stained the infected cells using red or orange-red fluorescence Annexin V PE/7-aminoactinomycin D to label the infected cells in the different apoptotic stages, and data acquisition was achieved by FCM $\left({ }^{*} \mathrm{p}<0.05 ;{ }^{* *} \mathrm{p}<0.01\right.$, compared with the pGC-FUGFP-infected cells).

applied. Western blot analysis showed that p73 and Wwox in the precipitation liquid harvested by co-immunoprecipitation (Wwox-agarose A/G) from pGC-FU-WWOX-infected cells were both present. At the same time, the mutual detection also showed that Wwox and p73 in the precipitation liquid harvested by co-immunoprecipitation (p73-agarose $\mathrm{A} / \mathrm{G}$ ) from the $\mathrm{pGC}$ FU-WWOX-infected cells were both also detectable. p53 for both Jurkat and $\mathrm{K} 562$ cells was undetectable in this process (Fig. 5).

\section{Discussion}

In the present study, we tranfected WWOX cDNA into human Jurkat and K562 leukemia cell lines using the lentiviral vector pGC-FU-WWOX, and we explored the effects of WWOX overexpression on the biological properties of these cell lines. Our data revealed that WWOX overexpression resulted in significant supression of cell viability and apoptosis induction in the Jurkat and K562 cells. We also investigated whether Wwox interacts with p73 or p53 in regards to its proapoptotic activity. We found that WWOX binds with p73 instead of p53 in the cytoplasm, indicating that WWOX has a close relationship with p73 during its proapoptotic activity in human leukemia.

Reduced WWOX expression and/or aberrant WWOX mRNA transcripts have been reported in various types of solid cancers (19-21), and restoration or upregulation of WWOX in tumor cell lines such as lung, breast and prostate, can sensitize them to apoptosis $(11,12,22)$, suggesting that WWOX functions as a tumor-suppressor gene. WWOX also plays an important role in human hematopoietic malignancies, as aberration or absence of WWOX expression has been detected in primary hematopoietic malignancies $(23,24)$. In the present study, we successfully transfected WWOX cDNA into Jurkat and K562 cells and observed overexpression of WWOX that resulted in marked inhibition of cell viability and promotion of apoptosis.
Although opposing views on the function of WWOX as a tumor-suppressor gene exist (25), the functional concept that ectopic expression of WWOX leads to apoptosis in leukemia cells was investigated in our study.

WWOX is reported to have a close relationship with the tumor-suppressor genes p73 and p53 (14-18,26,27). The first partner of the WW domain of Wwox to be reported was the p53 homologue named p73, and Wwox interacts via its first WW domain with the proline-rich motif of p73 (14). Of note, in a review by Chang, it was reported that suppression of WWOX expression abolishes p53-mediated apoptotic function, indicating that WWOX is a likely partner of p53 in cell apoptosis (26). Gomes et al also found that WWOX has a close relationship with p53 (18). However, studies by Aqeilan et al showed that WWOX is able to interact with $\mathrm{p} 73$ and suppresses its transcriptional activity $(14,27)$.

Our data demonstrated that Wwox is able to bind with cytoplasmic p73 instead of p53, without causing a nuclearplasma translocation for p73 in Jurkat and K562 cells. Aqeilan et al (14) reported that p73 localizes in the nucleus of NIH 3 T3 cells, while Wwox localizes to the cytoplasm, and overexpression of WWOX caused the sequestration of p73 from the nucleus to the cytoplasm in NIH $3 \mathrm{~T} 3$ cells; at the same time, cytoplasmic p73 contributed to the proapoptotic activity of Wwox.

Another review reported that WWOX and p53 both co-localize to the cytoplasm, and Wwox binds to the prolinerich region of p53 via its WW domain (16). Our findings do not agree with the viewpoints of a previous study (26) and do not agree in part with another study (14), as we did not observe a re-localization of $\mathrm{p} 73$ from the nucleus to the cytoplasm or from the cytoplasm to the nucleus in the pGCFU-WWOX-infected cells, as well as interactions between Wwox and p53. Yet, our findings are supported in part and fully by previous studies $(14,27)$. 
$\mathbf{A}$
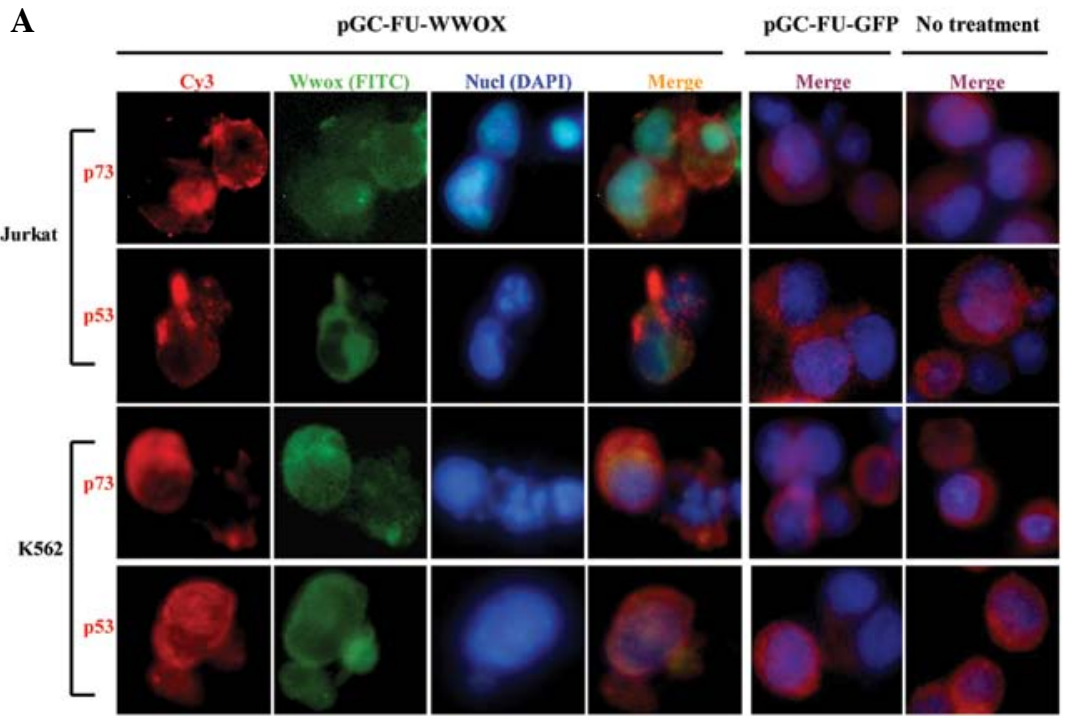

$\mathbf{B}$
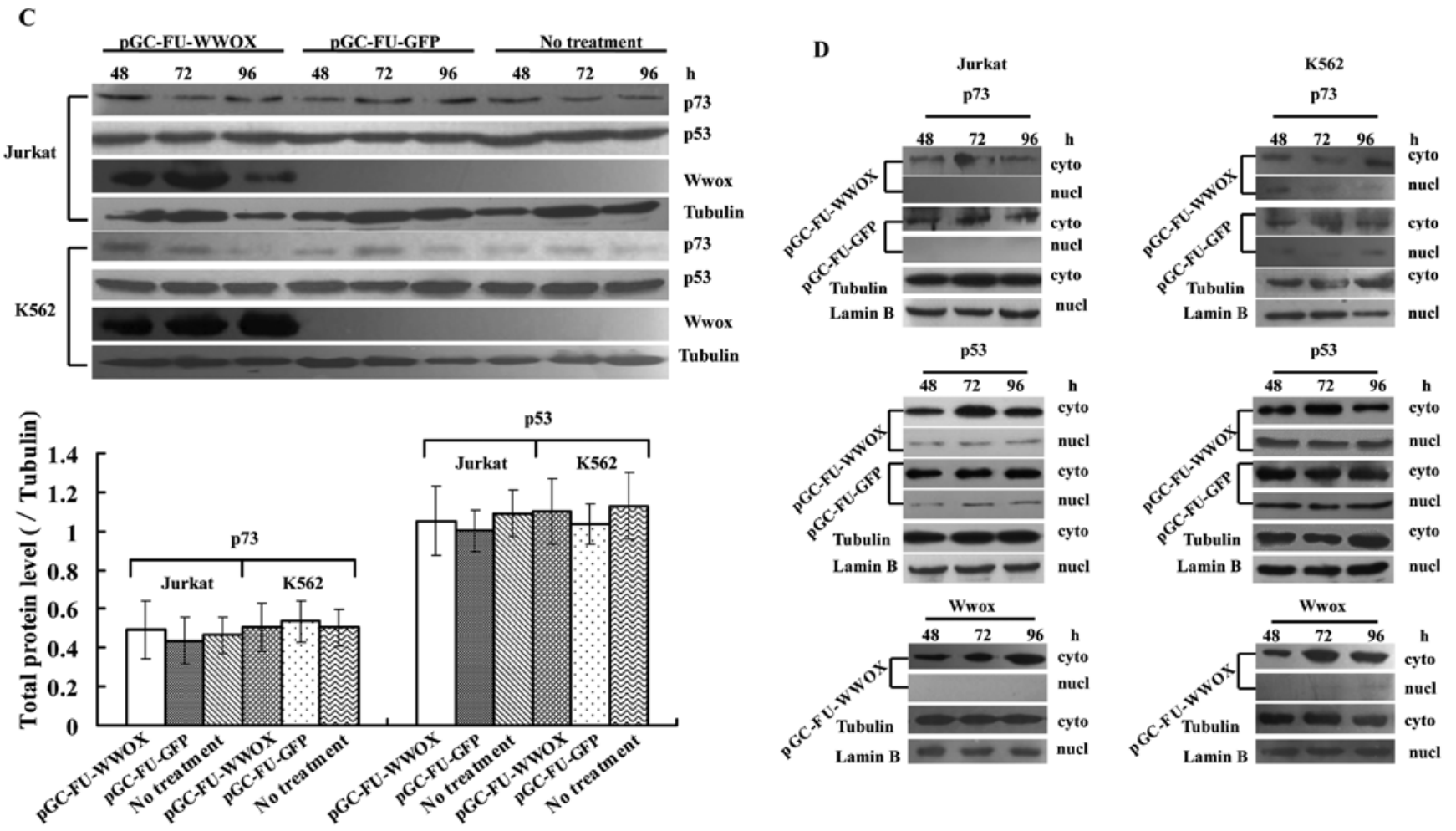

Figure 4. WWOX overexpression does not cause a change or dislocation of p73 and p53 in Jurkat or K562 cells as measured by immunofluorescence assay, qPCR and western blotting. (A) Subcellular locations of Wwox, p73 and p53 in pGC-FU-WWOX- and pGC-FU-GFP-infected cells. Immunofluorescence assay (observed by phase-contrast fluorescence microscopy at $60 \mathrm{~h}$ after infection; $\mathrm{x} 400$ ). As GFP is easily destroyed by the denaturant while staining, green fluorescence FITC-conjugated anti-rabbit IgG was used to label Wwox; p53 and p73 were labeled by red fluorescence Cy3-conjugated anti-mouse IgG, and the nucleus was dyed by DAPI. Changes in p73 or p53 are indicated by (B) qPCR and (C and D) western blot analysis [(C) total protein for p73 and p53; (D) protein in different organelles for p73 and p53. cyto, cytoplasm; nucl, nucleus].

One of the possible explanations is that the translocation of p73 or p53 in different types of cell lines is inherently different. For instance, p73 mainly localizes in the cytoplasm of Jurkat and K562 cells, and the nucleus exhibits p53 to a small extent, while in NIH 3T3 cells, p73 exhibits a high expression level in the nucleus (14). However, in our study we observed that Wwox and p73 co-localize in the cytoplasm of both Jurkat and K562 cells and bind together. Although p53 was not observed to bind with Wwox in our experiment, it is not proved that they have no interactions, and may probably exist in another manner.
Notably, we found a high expression level of p53 in both cell lines although they were between passages 15 and 30 . The most likely explanation is that the p53 protein was not encoded by the wild-types, but the mutant ones, which indicated that there might exist mutations of p53 in Jurkat and K562 cells. Nevertheless, it is unclear whether the p53 protein in our samples was wild-type or not, and more evidence is needed.

In summary, this is the first study to upregulate Wwox expression in human leukemia, in order to uncover the preliminary mechanisms of WWOX-mediated apoptosis in Jurkat and 


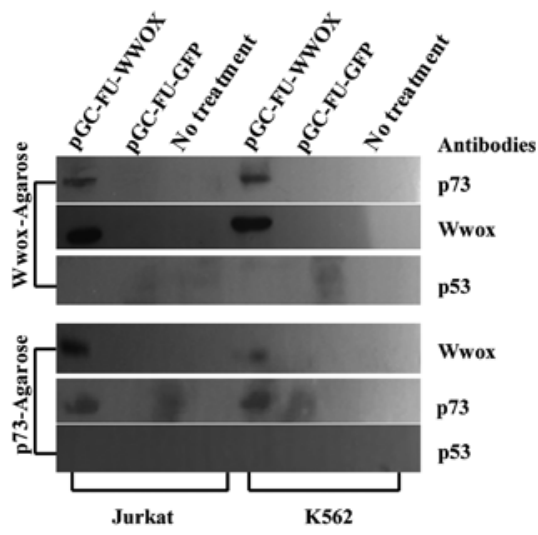

Figure 5. Interactions between Wwox and p73. For the co-immunoprecipitation assay, mutual detection was performed by using Wwox-agarose A/G to hook $\mathrm{p} 73$ or $\mathrm{p} 53$, and $\mathrm{p} 73$-agarose $\mathrm{A} / \mathrm{G}$ to hook Wwox or $\mathrm{p} 53$. The liquid harvested in this process was measured by western blotting.

K562 cells. We demonstrated that WWOX has a close relationship with p73 in its proapoptotic activity in leukemia. However, our study did not trace the actual binding sites between WWOX and p73, as well as the phosphorylated levels for p73 and p53 expression. Furthermore, a mammalian two hybrid assay or GST-pull down analysis is needed. These experiments will be performed in future studies by our group.

\section{Acknowledgements}

This study was supported by the Provincial Natural Science Fund of Fujian, grant no. 2010J01181.

\section{References}

1. Bednarek AK, Laflin KJ, Daniel RL, Liao Q, Hawkins KA and Aldaz CM: WWOX, a novel WW domain-containing protein mapping to human chromosome $16 \mathrm{q} 23.3-24.1$, a region frequently affected in breast cancer. Cancer Res 60: 2140-2145, 2000.

2. Ried K, Finnis M, Hobson L, et al: Common chromosomal fragile site FRA16D sequence: identification of the FOR gene spanning FRA16D and homozygous deletions and translocation breakpoints in cancer cells. Hum Mol Genet 9: 1651-1663, 2000.

3. Del Mare S, Salah Z and Aqeilan RI: WWOX: its genomics, partners, and functions. J Cell Biochem 108: 737-745, 2009.

4. Macias MJ, Wiesner S and Sudol M: WW and SH3 domains, two different scaffolds to recognize proline-rich ligands. FEBS Lett 513: 30-37, 2002.

5. Nunez MI, Ludes-Meyers $\mathbf{J}$ and Aldaz CM: WWOX protein expression in normal human tissues. J Mol Hist 37: 115-125, 2006

6. Wang X, Chao L, Jin G, Ma G, Zang Y and Sun J: Association between $\mathrm{CpG}$ island methylation of the WWOX gene and its expression in breast cancers. Tumour Biol 30: 8-14, 2009.

7. Guo W, Wang G, Dong Y, Guo Y, Kuang G and Dong Z: Decreased expression of WWOX in the development of esophageal squamous cell carcinoma. Mol Carcinog: Dec. 27, 2011 (Epub ahead of print).
8. Nakayama S, Semba S, Maeda N, Matsushita M, Kuroda Y and Yokozaki H: Hypermethylation-mediated reduction of WWOX expression in intraductal papillary mucinous neoplasms of the pancreas. Br J Cancer 100: 1438-1443, 2009.

9. Dias EP, Pimenta FJ, Sarquis MS, et al: Association between decreased WWOX protein expression and thyroid cancer development. Thyroid 17: 1055-1059, 2007.

10. Yang J, Cogdell D, Yang D, et al: Deletion of the WWOX gene and frequent loss of its protein expression in human osteosarcoma. Cancer Lett 291: 31-38, 2010.

11. Fabbri M, Iliopoulos D, Trapasso F, et al: WWOX gene restoration prevents lung cancer growth in vitro and in vivo. Proc Natl Acad Sci USA 102: 15611-15616, 2005.

12. Iliopoulos D, Fabbri M, Druck T, Qin HR, Han SY and Huebner K: Inhibition of breast cancer cell growth in vitro and in vivo: effect of restoration of Wwox expression. Clin Cancer Res 13: 268-274, 2007.

13. Aqeilan RI, Trapasso F, Hussain S, et al: Targeted deletion of Wwox reveals a tumor-suppressor function. Proc Natl Acad Sci USA 104: 3949-3954, 2007.

14. Aqeilan RI, Pekarsky Y, Herrero JJ, et al: Functional association between Wwox tumor suppressor protein and p73, a p53 homolog. Proc Natl Acad Sci USA 101: 4401-4406, 2004.

15. Hezova R, Ehrmann J and Kolar Z: WWOX, a new potential tumor suppressor gene. Biomed Pap Med Fac Univ Palacky Olomouc Czech Repub 151: 11-15, 2007.

16. Yang JL and Zhang W: WWOX tumor suppressor gene. Histol Histopathol 23: 877-882, 2008

17. Chang NS, Hsu LJ, Lin YS, Lai FJ and Sheu HM: WW domaincontaining oxidoreductase: a candidate tumor suppressor. Trends Mol Med 13: 12-22, 2007.

18. Gomes CC, Diniz MG, Oliveira CS, Tavassoli M, Odell EW, Gomez RS and De Marco L: Impact of WWOX alterations on $\mathrm{p} 73, \Delta \mathrm{Np} 73, \mathrm{p} 53$, cell proliferation and DNA ploidy in salivary gland neoplasms. Oral Dis 17: 564-571, 2011.

19. Aqeilan RI, Kuroki T, Pekarsky Y, et al: Loss of WWOX expression in gastric carcinoma. Clin Cancer Res 10: 3053-3058, 2004.

20. Alsop AE, Taylor K, Zhang J, Gabra H, Paige AJ and Edwards PA: Homozygous deletions may be markers of nearby heterozygous mutations: the complex deletion at FRA16D in the HCT116 colon cancer cell line removes exons of WWOX. Genes Chromosomes Cancer 47: 437-447, 2008.

21. Żelazowski MJ, Płuciennik E, Pasz-Walczak G, Potemski P, Kordek R and Bednarek AK: WWOX expression in colorectal cancer: a real-time quantitative RT-PCR study. Tumour Biol 32: 551-560, 2011.

22. Qin HR, Iliopoulos D, Semba S, et al: A role for the WWOX gene in prostate cancer. Cancer Res 66: 6477-6480, 2006.

23. Ishii $\mathrm{H}$ and Furukawa $\mathrm{Y}$ : Alterations of common chromosome fragile sites in hematopoietic malignancies. Int J Hematol 79: 238-242, 2004.

24. Ishii H, Vecchione A, Furukawa Y, et al: Expression of FRA16D/ WWOX and FRA3B/FHIT genes in hematopoietic malignancies. Mol Cancer Res 1: 940-947, 2003.

25. Watanabe A, Hippo Y, Taniguchi H, et al: An opposing view on WWOX protein function as a tumor suppressor. Cancer Res 63: 8629-8633, 2003.

26. Chang NS: A potential role of p53 and WOX1 in mitochondrial apoptosis (Review). Int J Mol Med 9: 19-24, 2002.

27. Aqeilan RI, Donati V, Palamarchuk A, et al: WW domaincontaining proteins, WWOX and YAP, compete for interaction with ErbB-4 and modulate its transcriptional function. Cancer Res 65: 6764-6772, 2005. 